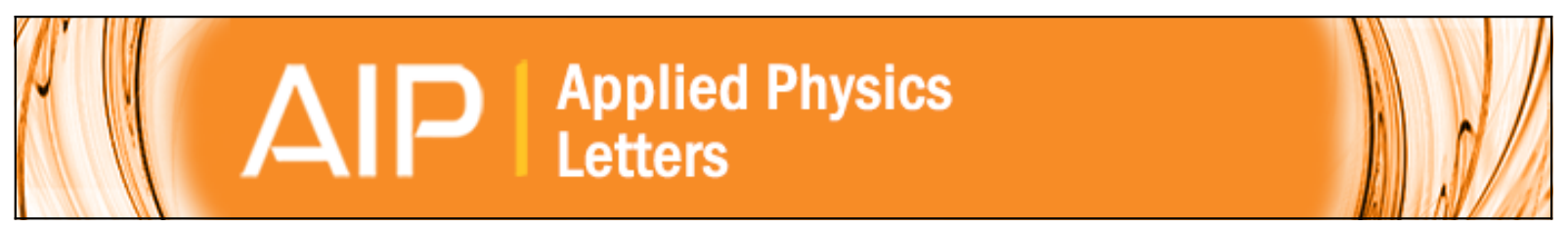

\title{
Perpendicular exchange bias effect in sputter-deposited CoFe/IrMn bilayers
}

J. Y. Chen, Naganivetha Thiyagarajah, H. J. Xu, and J. M. D. Coey

Citation: Applied Physics Letters 104, 152405 (2014); doi: 10.1063/1.4871711

View online: http://dx.doi.org/10.1063/1.4871711

View Table of Contents: http://scitation.aip.org/content/aip/journal/apl/104/15?ver=pdfcov

Published by the AIP Publishing

\section{Articles you may be interested in}

Effect of $\mathrm{Ga}+$ ion irradiation on the structural and magnetic properties of CoFe/lrMn exchange biased bilayers

J. Appl. Phys. 95, 7772 (2004); 10.1063/1.1745120

Exchange anisotropy and spin-wave damping in CoFe/lrMn bilayers

J. Appl. Phys. 93, 7717 (2003); 10.1063/1.1543126

Magnetization reversal of exchange coupled CoFe/lrMn bilayers

J. Appl. Phys. 91, 6890 (2002); 10.1063/1.1447185

Exchange bias measurements of CoFe//rMn

J. Appl. Phys. 89, 7543 (2001); 10.1063/1.1358832

CoFe//rMn exchange biased top, bottom, and dual spin valves

J. Appl. Phys. 87, 6989 (2000); 10.1063/1.372907

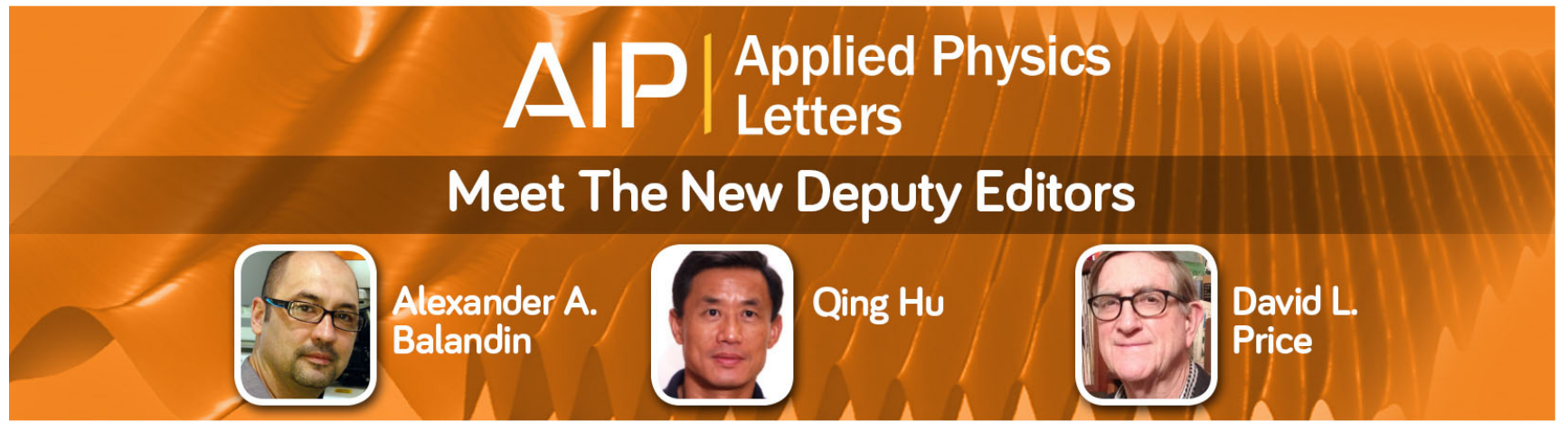




\title{
Perpendicular exchange bias effect in sputter-deposited CoFe/lrMn bilayers
}

\author{
J. Y. Chen, ${ }^{\text {a) }}$ Naganivetha Thiyagarajah, H. J. Xu, and J. M. D. Coey \\ School of Physics and CRANN, Trinity College, Dublin 2, Ireland
}

(Received 23 January 2014; accepted 6 April 2014; published online 16 April 2014)

\begin{abstract}
$\mathrm{CoFe} / \mathrm{IrMn}$ bilayers with perpendicular magnetization for various IrMn layer thicknesses exhibit unusual two-step hysteresis loops with both positive and negative loop shifts. Observed at room temperature in the as-grown state, they provide direct evidence of large antiferromagnetic domain formation at the IrMn interface. The exchange bias field reaches $100 \mathrm{mT}$ with an $\mathrm{IrMn}$ layer thickness of $4 \mathrm{~nm}$ after field annealing at $200{ }^{\circ} \mathrm{C}-300^{\circ} \mathrm{C}$ in $800 \mathrm{mT}$, which is at least three times as large as the coercivity, and may be useful for reference layers of spin-valves or magnetic tunnel junctions with perpendicular magnetic anisotropy. (C) 2014 AIP Publishing LLC. [http://dx.doi.org/10.1063/1.4871711]
\end{abstract}

Exchange bias, created at the interface between an antiferromagnetic (AFM) and a ferromagnetic (FM) material, is manifest as a shift in the hysteresis loop and an enhancement of coercivity when a composite structure is field-cooled through the Néel temperature $\left(T_{N}\right)$ of the AFM material. ${ }^{1,2}$ Despite extensive research over more than half a century, the microscopic origin is still not fully understood, although it is generally accepted that the effect results from exchange coupling between FM and uncompensated AFM spins at the interface. As a result, it is very sensitive to the interfacial spin structure. The exchange bias effect in bilayers with inplane anisotropy has been widely used in magnetic devices such as sensors and magnetic random access memory (MRAM) ${ }^{3-7}$ which are based on spin valves or magnetic tunnel junctions (MTJs).

More recently, there has been an increasing interest in exchange bias of materials with perpendicular magnetic anisotropy. Perpendicular exchange bias (PEB) has been observed in $\mathrm{Co}(\mathrm{CoFe}) / \mathrm{Pt}(\mathrm{Pd})$ multilayers coupled with an AFM material such as $\mathrm{CoO}, \mathrm{NiO}, \mathrm{FeMn}$, or $\mathrm{IrMn}^{8-14}$ However, most of the reported values for the PEB field are at best comparable to the coercive field and may be too weak for practical applications. Many researchers have tried to improve the PEB using different methods. For example, Liu et al.$^{15}$ obtained a high PEB field of about $95 \mathrm{mT}$ by inserting a CoFe layer between $\mathrm{IrMn}$ and $\mathrm{Co} / \mathrm{Pt}$ multilayers at room temperature. Wang et al. ${ }^{16}$ reported a large PEB effect in $\mathrm{CoPt} / \mathrm{CoO}$ multilayers at low temperature. The interfacial spin structure at the FM/AFM interface is thought to be crucial for PEB. While these studies showed large effects, the thermal stability and magnetic properties, which are important for device applications, were not fully investigated. Most reports in the literature have focused on $\mathrm{FM} / \mathrm{Pt}(\mathrm{Pd})$ multilayers, which often exhibit poor thermal stability due to interface diffusion. FM/AFM bilayers are thought to have better thermal stability, as the interface diffusion is reduced. Furthermore, it is easier to identify the interface behavior, as the contribution of other layers is absent. Ultrathin ferromagnetic layers are known to exhibit perpendicular magnetic

\footnotetext{
${ }^{\text {a) }}$ Author to whom correspondence should be addressed. Electronic mail: chenjy02@gmail.com
}

anisotropy ${ }^{17}$ but there are only limited studies of PEB in FM/AFM bilayers. ${ }^{18,19}$

Here, we discuss PEB in a simple bilayer structure of ultrathin $\mathrm{CoFe}$ and IrMn, where anomalous two-step hysteresis loops with both positive and negative loop shifts are observed in as-grown samples.

The samples were deposited onto thermally oxidized $\mathrm{Si}$ wafers by DC magnetron sputtering at room temperature in a Shamrock deposition tool. The stacks were (from bottom to top) $\mathrm{Ta} 5 / \mathrm{Pt} 5 / \mathrm{Co}_{90} \mathrm{Fe}_{10}(\mathrm{CoFe}) 0.8 / \mathrm{Ir}_{22} \mathrm{Mn}_{78}(\mathrm{IrMn})\left(t_{\text {IrMn }}\right) / \mathrm{Pt}$ 2 (thickness in $\mathrm{nm}$ ). A single ultrathin top-pinned CoFe layer with a thickness of $0.8 \mathrm{~nm}$ grown on a buffer of $\mathrm{Ta} / \mathrm{Pt}$ showed good perpendicular magnetic anisotropy. The thickness of the IrMn layer $\left(t_{I r M n}\right)$ was varied from $0 \mathrm{~nm}$ to $12 \mathrm{~nm}$. Samples were post-deposition annealed for $1 \mathrm{~h}$ at temperatures ranging from $200^{\circ} \mathrm{C}$ to $300^{\circ} \mathrm{C}$ in an applied magnetic field of $800 \mathrm{mT}$. The field was perpendicular to the film surface. Out-of-plane magnetic hysteresis loops were measured using the extraordinary Hall Effect (EHE) on $6 \times 6 \mathrm{~mm}^{2}$ samples, contacted at the four corners in the Van der Pauw geometry. The thickness, interface roughness, and the crystal structure were characterized by using X-ray Reflectivity (XRR) and X-ray Diffraction (XRD). The microstructure of the films was also characterized by high-resolution transmission electron microscopy (TEM). Cross-section lamellas of the films for TEM observation were prepared by focused ion beam (FIB). Magnetic domains were imaged by magnetic force microscopy (MFM), using a Nanoscope III instrument with a low moment tip.

Figures 1(a) and 1(b) show the typical XRR and XRD patterns for the as-grown $\mathrm{CoFe} / \mathrm{IrMn}$ bilayers with different thicknesses of IrMn, $t_{I r M n}=4,6$, and $10 \mathrm{~nm}$. Very clear XRR oscillations are observed for all the samples, as seen in Fig. 1(a). Well-defined interfaces are needed to produce such oscillations. The thickness and interface roughness for each layer are obtained by fitting the curves using the WinGixa software package. The fitted CoFe layer thickness lies in the range of $0.72-0.82 \mathrm{~nm}$ for all the samples, which is close to the nominal value. The roughness of both FM/AFM and $\mathrm{FM} /$ buffer interfaces is $0.3-0.5 \mathrm{~nm}$, which suggests that the $\mathrm{CoFe}$ layer grows as a continuous film, as also seen in the cross-section TEM images (Fig. 2). From the XRD patterns in Fig. 1(b), it can be seen that all the samples show strong fcc Pt (111) and fcc IrMn (111) texture. An evident bcc Ta 

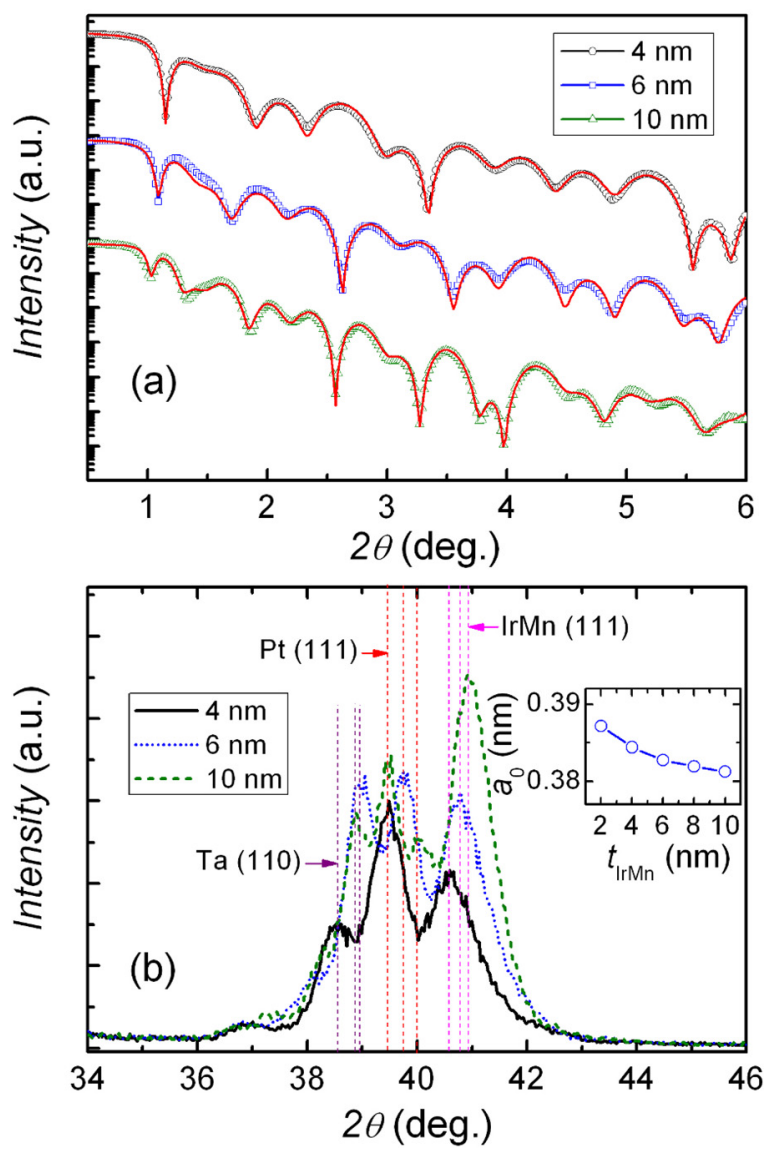

FIG. 1. The typical (a) XRR and (b) XRD patterns for the as-grown $\mathrm{CoFe} / \mathrm{IrMn}$ bilayers with different thicknesses of IrMn layer. The inset of (b) gives the lattice constant of the IrMn layers.

(110) peak is also observed. Due to the ultrathin nature of the $\mathrm{CoFe}$ ferromagnetic layer, no crystalline peaks are observable; however, the perpendicular anisotropy is induced due to the interfacial anisotropy at the $\mathrm{CoFe} / \mathrm{Pt}$ (111) interface. In addition, the strong (111) texture of the antiferromagnetic fcc IrMn layer is the structure necessary to induce PEB in this system. Almost all peaks are shifted compared to the bulk materials, which are thought to result from mechanically induced strain during deposition. The lattice constant $\left(a_{0}\right)$ for the IrMn layers, calculated from (111) reflection, is found to shrink from $0.387 \pm 0.001 \mathrm{~nm}$ $\left(t_{\text {IrMn }}=2 \mathrm{~nm}\right)$ to $0.381 \pm 0.001 \mathrm{~nm}\left(t_{\text {IrMn }}=10 \mathrm{~nm}\right)$ with the increase of $t_{I r M n}$, as shown in the inset of Fig. 1(b), approaching the bulk value $\left(a_{0}=0.378 \mathrm{~nm}\right)$. The variation of the IrMn lattice constant indicates that the strain induced by the crystal lattice in the IrMn layer is gradually relaxed by the formation of dislocations.

The out-of-plane magnetic properties of $\mathrm{CoFe} / \mathrm{IrMn}$ bilayers with various $t_{I r M n}$ are measured using EHE, as shown in Figs. 3(a) and 3(b). When $t_{I r M n}$ is below $2 \mathrm{~nm}$, the loops are almost perfectly square, indicating that the $\mathrm{CoFe}$ nanolayer has perpendicular magnetic anisotropy. However, no PEB effects are observed. When $t_{I r M n}$ is increased to $3 \mathrm{~nm}$, the PEB effect appears, and the system exhibits maximum coercivity $\left(\mu_{0} H_{c}=98.2 \mathrm{mT}\right)$. There are unusual two-step EHE loops with both positive and negative loop shifts when $t_{I r M n} \geq 3 \mathrm{~nm}$. The PEB fields $\left(\mu_{0} H_{e x+}\right.$ for the positive part, $\mu_{0} H_{e x-}$ for negative part) and coercivities $\left(\mu_{0} H_{c+}\right.$

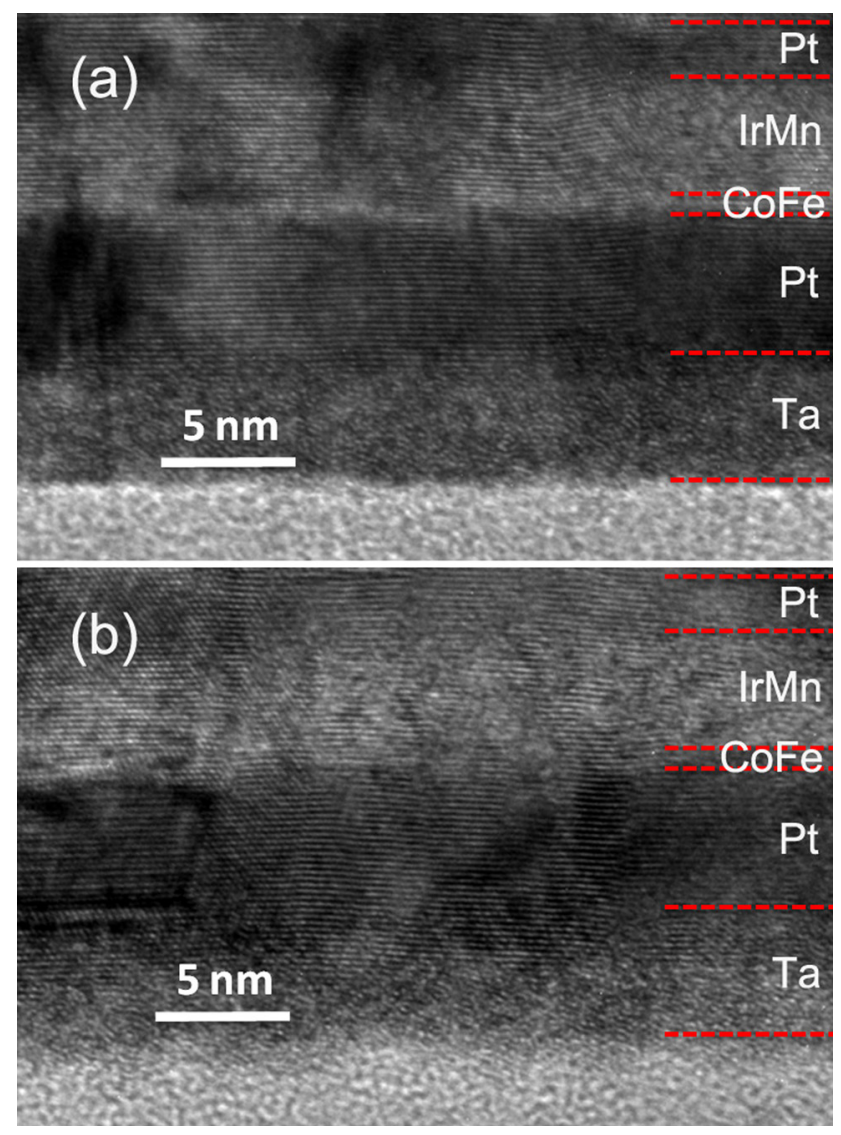

FIG. 2. TEM cross-section of a CoFe/IrMn bilayer with nominal thickness $0.8 / 4 \mathrm{~nm}$ (a) for as-grown state and (b) after annealing for $1 \mathrm{~h}$ at $200^{\circ} \mathrm{C}$ under a field of $800 \mathrm{mT}$.

for positive part, $\mu_{0} H_{c-}$ for negative part) as a function of $t_{I r M n}$ are summarized in Fig. 3(c). The highest $\mu_{0} H_{e x+}$ $\left(\mu_{0} H_{e x-}\right)$ of $91.3 \mathrm{mT}(-86.1 \mathrm{mT})$ has been obtained at as-grown state with $t_{I r M n}=4 \mathrm{~nm}$. With further increase of $t_{\text {IrMn }}$ to $12 \mathrm{~nm}, \mu_{0} H_{e x+}\left(\mu_{0} H_{e x-}\right)$ remains almost constant.

According to Meiklejohn and Bean's model, ${ }^{1,2}$

$$
\mu_{0} H_{e x}=\frac{\sigma_{e x}}{M_{F M} t_{F M}},
$$

where $\sigma_{e x}$ is the interfacial exchange coupling, $M_{F M}$ is the magnetization of the FM layer, and $t_{F M}$ is the thickness of the FM layer. In our case, we kept the same CoFe layer thickness for all the samples, so, $M_{F M} t_{F M}$ is constant. Hence, we deduce $\sigma_{e x}=0.11 \mathrm{~mJ} / \mathrm{m}^{2}$, which is comparable to the exchange coupling for the in-plane system. The IrMn layer is polycrystalline with a preferred fcc (111) texture. It is expected to be chemically disordered with the Ir and Mn atoms occupying the fcc atomic-sites at random in the $\mathrm{IrMn}_{3}$ structure. The magnetic moments are tilted away by $45^{\circ}$ from the $\{111\}$ diagonals towards the cube faces. ${ }^{20}$ We can consider that the polycrystalline IrMn layer has uncompensated spins and is composed of tiny AFM grains with each contributing to the PEB effect. ${ }^{21}$ For a single AFM grain, $\sigma_{e x} \propto A / D$, where $A$ is interfacial exchange stiffness and $D$ is the AFM grain size. ${ }^{22}$ The AFM grains do not contribute to the loop shift below a critical thickness $t_{A F M}=\sigma_{e x} / K_{A F M}$, where $K_{A F M}$ is uniaxial anisotropy constant of the antiferromagnet. The critical $t_{A F M}=3 \mathrm{~nm}$ gives $K_{A F M}=3.7 \times 10^{4} \mathrm{~J} / \mathrm{m}^{3}$ for IrMn layer. The 

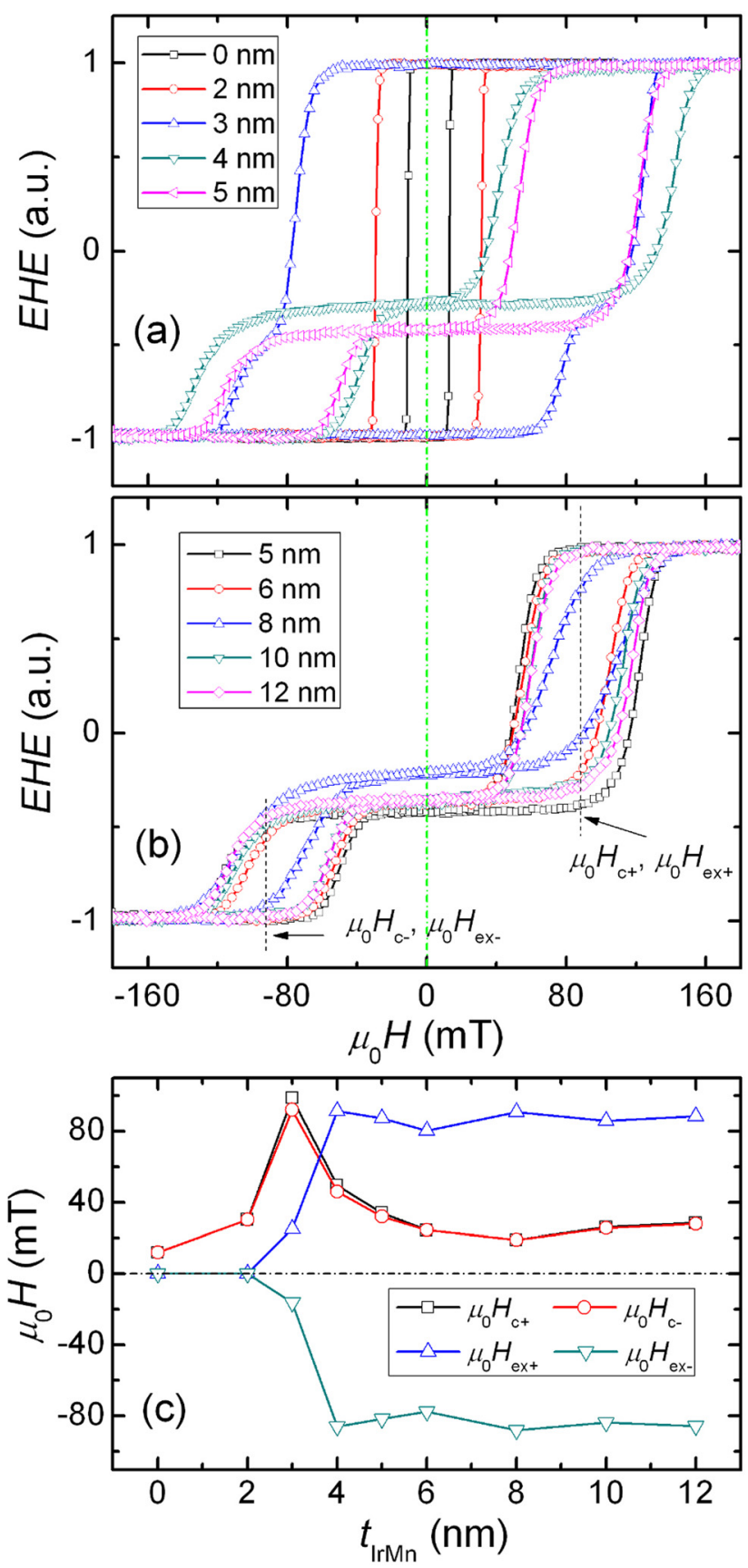

FIG. 3. (a) and (b) Out-of-plane EHE hysteresis loops of as-grown CoFe/IrMn bilayers with different thicknesses of IrMn layers. (c) Perpendicular exchange bias field $\left(H_{e x}\right)$ and coercivity $\left(H_{c}\right)$ as a function of $t_{I r M n}$.

critical AFM grain size, above which PEB is obtained, is given as $D_{C}=A / K_{A F M} t_{A F M}$. The smaller $t_{A F M}$, the larger the critical grain size, and the average grain size scales with $t_{A F M}$. This explains why we do not observe any PEB effect when $t_{I r M n}$ is below $2 \mathrm{~nm}$, which is consistent with other reports. ${ }^{23,24}$ When $t_{I r M n}$ is increased from $2 \mathrm{~nm}$ to $4 \mathrm{~nm}$, it may be assumed that many of the grains have $D>D_{c}$, and $H_{e x}$ quickly increases to the maximum value. ${ }^{22}$ Further increase in $t_{I r M n}$ up to $12 \mathrm{~nm}$ does not significantly change $H_{e x}$, because $\sigma_{e x}$ remains similar.

Figures 4(a)-4(f) show the magnetic domain patterns in the as-grown remnant state with different thicknesses of the IrMn layer, $t_{I r M n}$ ranging from $2 \mathrm{~nm}$ to $12 \mathrm{~nm}$. In our $\mathrm{CoFe} / \mathrm{IrMn}$ bilayers, the $\mathrm{IrMn}$ is grown directly on top of the
CoFe layer, and spins in the IrMn layer will couple to spins in the adjacent $\mathrm{CoFe}$ layer. There are no visible domains, when $t_{I r M n} \leq 2 \mathrm{~nm}$, which means that our perpendicular $\mathrm{CoFe}$ layer is essentially in a single perpendicular domain state. The interface exchange coupling from the IrMn is quite weak and has no influence on the ferromagnetic domain structures. Moreover, when $t_{I r M n} \geq 3 \mathrm{~nm}$, the interface exchange coupling with IrMn becomes sufficient strong for exchange bias to appear, and the bilayer exhibits a multidomain state, illustrated in Figs. 4(b)-4(f). This reflects the magnetic structure of the IrMn layer at the interface, to which the $\mathrm{CoFe}$ is coupled. We are therefore imaging the AFM multidomain state of the IrMn. All these bilayers exhibit similar domains. The multigrain antiferromagnetic domains are large, with dimension of approximate $10 \times 1 \mu \mathrm{m}^{2}$. The unusual two-step magnetization reversal with both positive and negative loop shifts, shown in Figs. 3(b) and 3(c), is a consequence of formation of large antiferromagnetic domains in the as-grown state. Although the IrMn grain size is only of order $10 \mathrm{~nm}$, the layer is strongly textured, and multigrain domains are anticipated, as with nanoscale ferromagnets. ${ }^{25}$ Similar behavior has been reported in other exchange-biased $\mathrm{NiFe} / \mathrm{FeMn}$ and $\mathrm{NiCo} / \mathrm{FeMn}$ bilayers with in-plane anisotropy. ${ }^{26,27}$ The coercive fields, $\mu_{0} H_{c+}$ and $\mu_{0} H_{c-}$, of both the positive and negative loops are almost same, and $\mu_{0} H_{e x+}$ and $\mu_{0} H_{e x-}$ are symmetric, as evidenced by the up and down domains in the MFM image.

The magnetic field annealing performed in $800 \mathrm{mT}$ for these $\mathrm{CoFe} / \mathrm{IrMn}$ bilayers sets a unique direction of exchange bias. Fig. 5(a) shows typical EHE hysteresis loops for the sample with $t_{I r M n}=4 \mathrm{~nm}$ with and without magnetic field annealing. After annealing at $200^{\circ} \mathrm{C}$, the two-step EHE loop disappears. The $\mathrm{CoFe} / \mathrm{IrMn}$ bilayers exhibit a single-step EHE loop with negative PEB. It suggests that all the uncompensated spins at the interface for the tiny IrMn grains have been aligned to the direction of magnetic field and a single FM domain state is formed. We also annealed the $\mathrm{CoFe} / \mathrm{IrMn}$ bilayers under the same magnetic field applied in both the positive $(+)$ and negative $(-)$ directions, and as shown in the inset of Fig. 5(a), either a negative or a positive EHE loop shift with PEB field of almost same value results. The highest PEB field, $\mu_{0} H_{e x}$, that has been found after annealing is $100.5 \mathrm{mT}$ (Fig. 5(a)), which is the largest value reported in this system so far. On annealing the sample at higher temperatures, the perpendicular magnetic anisotropy gradually decreases, but the PEB field remains almost the same $\left(\mu_{0} H_{e x}=100.5 \pm 0.8 \mathrm{mT}\right)$, as shown in Fig. 5(a). In addition, the PEB field is much larger than $H_{c}$ (typically, $\mu_{0} H_{c}=38.3 \mathrm{mT}$ at $200^{\circ} \mathrm{C} ; \quad \mu_{0} H_{c}=29.2 \mathrm{mT}$ at $250^{\circ} \mathrm{C}$; $\mu_{0} H_{c}=14.6 \mathrm{mT}$ at $300^{\circ} \mathrm{C}$ ). This feature makes it favorable to integrate a $\mathrm{CoFe} / \mathrm{IrMn}$ bilayer system into the reference layer of perpendicular spin-valve or MTJ-based MRAM, so that the reference layer is more stable under the writing field/current. The decrease of perpendicular magnetic anisotropy after annealing is attributed to reduction of interface anisotropy due to interfacial diffusion at the $\mathrm{CoFe} / \mathrm{Pt}$ and $\mathrm{CoFe} / \mathrm{IrMn}$ interfaces (Fig. 2(b)). The $t_{I r M n}$ dependence of $\mu_{0} H_{e x}$ and $\mu_{0} H_{c}$ with field annealing is shown in Fig. 5(b). Unlike the case in the as-grown state, as $t_{I r M n}$ increases from $4 \mathrm{~nm}$ to $12 \mathrm{~nm}, \mu_{0} H_{e x}$ gradually decreases after field 

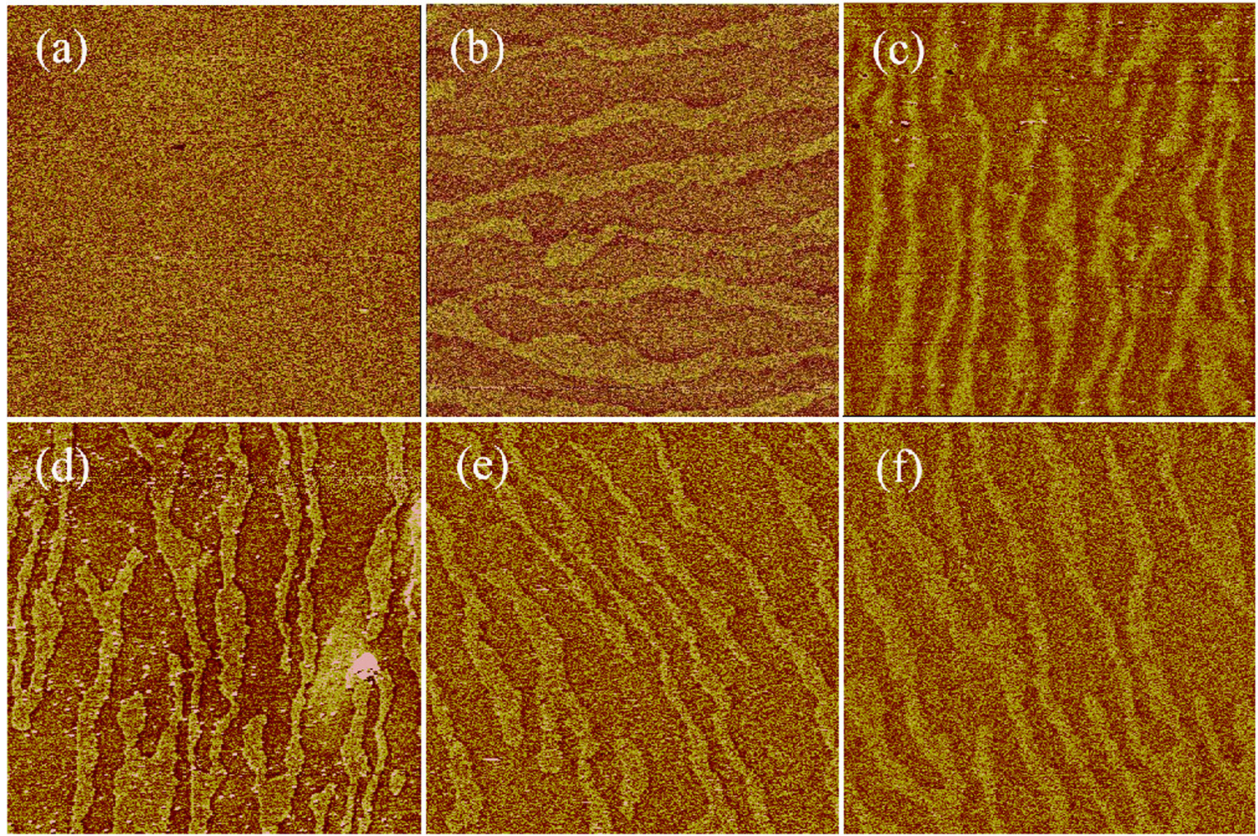

FIG. 4. MFM images with size of $10 \times 10 \mu \mathrm{m}^{2}$ taken for the bilayers with different IrMn layer thicknesses $\left(t_{I r M n}\right)$ in the as-grown remnant state. Typically, (a) $t_{I r M n}=2 \mathrm{~nm}$; (b) $t_{I r M n}=3 \mathrm{~nm}$; (c) $t_{I r M n}=4 \mathrm{~nm}$; (d) $t_{I r M n}=6 \mathrm{~nm}$; (e) $t_{I r M n}=10 \mathrm{~nm}$; and (f) $t_{I r M n}=12 \mathrm{~nm}$.

annealing. The main reason may be that $H_{c}$ and the perpendicular magnetic anisotropy gradually degrade after field annealing the thicker IrMn layer, leading to a decrease of the magnetization of the CoFe. According to Eq. (1), the
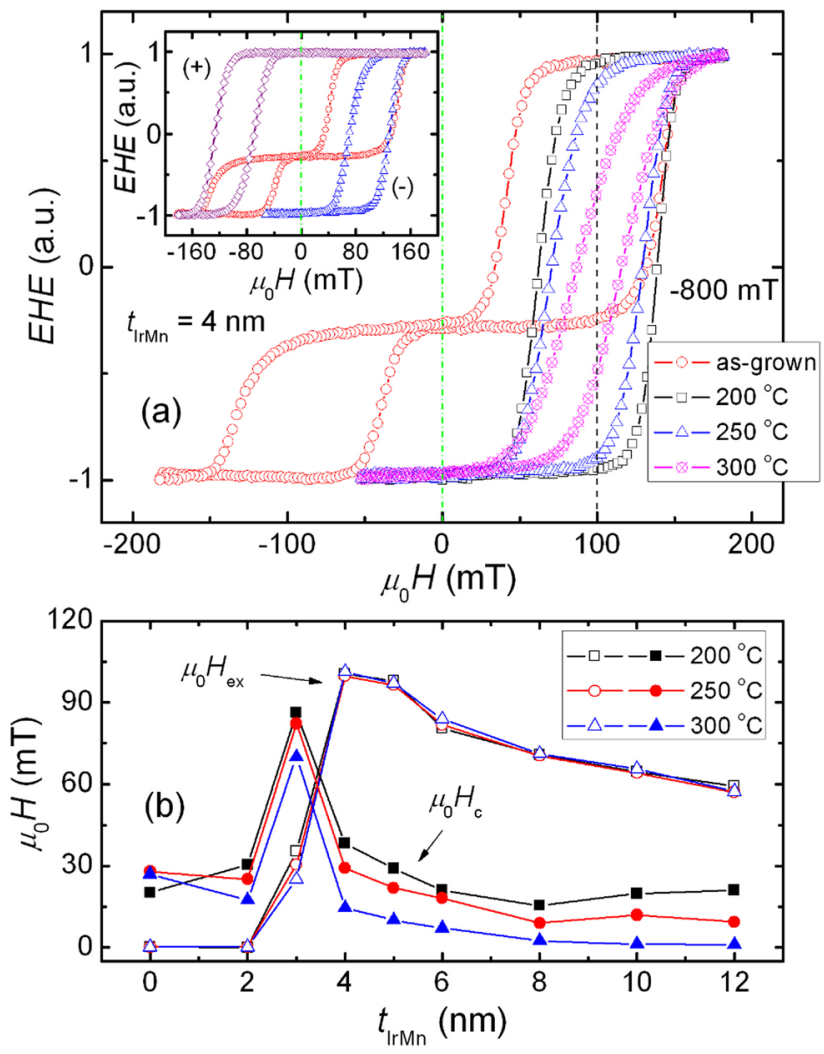

FIG. 5. (a) Out-of-plane EHE hysteresis loops at different annealing temperatures for the CoFe/IrMn bilayers with IrMn layer thickness of $4 \mathrm{~nm}$ under an annealing field of $-800 \mathrm{mT}$. The inset shows obtained negative and positive EHE loops shifts under positive $(+)$ or negative $(-)$ field annealing at $250^{\circ} \mathrm{C}$. (b) Perpendicular exchange bias field $\left(H_{e x}\right)$ and coercivity $\left(H_{c}\right)$ as a function of $t_{I r M n}$ under different annealing temperatures. reduction in $\mu_{0} H_{e x}$ indicates that the exchange coupling constant $\sigma_{e x}$ is weak. The smaller the $t_{I r M n}$ (above the critical thickness), the better the thermal stability.

In conclusion, we have demonstrated a robust PEB effect in $\mathrm{CoFe} / \mathrm{IrMn}$ bilayers as a function of IrMn layer thicknesses and annealing. The anomalous two-step hysteresis loops with both positive and negative loop shifts observed in the as-grown state when $t_{I r M n} \geq 3 \mathrm{~nm}$ are direct evidence for the formation of large AFM domains at the interface, consisting of many IrMn grains. After magnetic field annealing, the two-step magnetization reversal disappears and either positive or negative shifted loops with one-step magnetization reversal are observed, depending on the magnetic field direction. The PEB field of about $100 \mathrm{mT}$ is the highest reported in this kind of system, and the effect could be usefully applied in perpendicular spin valves or magnetic tunnel junctions.

The authors thank Clive Downing at AML, Trinity College Dublin for the TEM. This work was supported by Science Foundation Ireland as part of work of the AMBER Center and the Nanoscale Interface and Spin Electronics (NISE) Project (10/IN1/I3006). Further support was provided by the EU as part of FP7 NAMDIATREAM Project.

${ }^{1}$ W. H. Meiklejohn and C. P. Bean, Phys. Rev. 102, 1413 (1956).

${ }^{2}$ W. H. Meiklejohn, J. Appl. Phys. 33, 1328 (1962).

${ }^{3}$ S. S. P. Parkin, K. P. Roche, M. G. Samant, P. M. Rice, R. B. Beyers, R. E. Scheuerlein, E. J. O'Sullivan, S. L. Brown, J. Bucchigano, D. W. Abraham, Y. Lu, M. Rooks, P. L. Trouilloud, R. A. Wanner, and W. J. Gallagher, J. Appl. Phys. 85, 5828 (1999).

${ }^{4}$ X. F. Han, Z. C. Wen, and H. X. Wei, J. Appl. Phys. 103, 07E933 (2008).

${ }^{5}$ P. P. Freitas, R. Ferreira, S. Cardoso, and F. Cardoso, J. Phys.: Condens. Matter 19, 165221 (2007).

${ }^{6}$ J. Y. Chen, J. F. Feng, and J. M. D. Coey, Appl. Phys. Lett. 100, 142407 (2012).

${ }^{7}$ J. Y. Chen, N. Carroll, J. F. Feng, and J. M. D. Coey, Appl. Phys. Lett. 101, 262402 (2012). 
${ }^{8}$ S. Matt, K. Takano, S. S. P. Parkin, and E. E. Fullerton, Phys. Rev. Lett. 87, 087202 (2001).

${ }^{9}$ J. Sort, V. Baltz, F. Garcia, B. Rodmacq, and B. Dieny, Phys. Rev. B 71, 054411 (2005).

${ }^{10}$ S. van. Dijken, J. Moritz, and J. M. D. Coey, J. Appl. Phys. 97, 063907 (2005).

${ }^{11}$ L. Lin, N. Thiyagarajah, H. W. Joo, J. Heo, K. A. Lee, and S. Bae, Appl. Phys. Lett. 97, 242514 (2010).

${ }^{12}$ Z. Y. Liu and S. Adenwalla, J. Appl. Phys. 94, 1105 (2003).

${ }^{13}$ J. Y. Chen, J. F. Feng, Z. Diao, G. Feng, J. M. D. Coey, and X. F. Han, IEEE Trans. Magn. 46, 1401 (2010).

${ }^{14}$ W. J. Gong, W. Liu, X. H. Liu, S. Guo, J. N. Feng, B. Li, and Z. D. Zhang, J. Appl. Phys. 109, 043906 (2011).

${ }^{15}$ Y. F. Liu, J. W. Cai, and S. L. He, J. Phys. D: Appl. Phys. 42, 115002 (2009).

${ }^{16}$ J. Wang, T. Omi, T. Sannomiya, S. Muraishi, J. Shi, and Y. Nakamura, Appl. Phys. Lett. 103, 042401 (2013).

${ }^{17}$ S. Ikeda, K. Miura, H. Yamamoto, K. Mizunuma, H. D, Gan, M. Endo, S. Kanai, J. Hayakawa, F. Matsukura, and H. Ohno, Nature Mater. 9, 721 (2010).
${ }^{18}$ Y. Shiratsuchi, H. Noutomi, H. Oikawa, T. Nakamura, M. Suzuki, T. Fujita, K. Arakawa, Y. Takechi, H. Mori, T. Kinoshita, M. Yamamoto, and R. Nakatani, Phys. Rev. Lett. 109, 077202 (2012).

${ }^{19}$ R. Yanes, J. Jackson, L. Udvardi, L. Szunyogh, and U. Nowak, Phys. Rev. Lett. 111, 217202 (2013).

${ }^{20}$ A. Kohn, A. Kavacs, R. Fan, G. J. Mclntyre, R. C. C. Ward, and J. P. Goff, Sci. Rep. 3, 2412 (2013).

${ }^{21}$ M. D. Stiles and R. D. McMichael, Phys. Rev. B 59, 3722 (1999).

${ }^{22}$ J. Moritz, G. Vinai, and B. Dieny, IEEE Magn. Lett. 3, 4000204 (2012).

${ }^{23}$ H. Xi and R. M. White, J. Appl. Phys. 94, 5850 (2003).

${ }^{24}$ H. Meng, V. B. Naik, and R. Sbiaa, Phys. Status Solidi A 210, 391 (2013).

${ }^{25}$ K. Khlopkov, O. Gutfleisch, D. Hinz, K. H. Muller, and L. Schultz, J. Appl. Phys. 102, 023912 (2007).

${ }^{26}$ C. L. Chien, V. S. Gornakov, V. I. Nikitenko, A. J. Shapiro, and R. D. Shull, Phys. Rev. B 68, 014418 (2003).

${ }^{27}$ J. Lin, Z. Shi, S. M. Zhou, X. Zhang, and Y. J. Xia, Chin. Phys. Lett. 26, 107501 (2009). 